\title{
Microwave synthesis and mechanical characterization of functionally graded material for applications in fusion devices
}

\author{
CHARU LATA DUBE ${ }^{\mathrm{a}, *}$, YASHASHRI PATIL ${ }^{\mathrm{a}}$, SHAILESH KANPARA ${ }^{\mathrm{a}}$, \\ SAMIR S KHIRWADKAR ${ }^{a}$ and SUBHASH C KASHYAP ${ }^{b}$ \\ ${ }^{a}$ Divertor \& Firstwall Technology Development Division, Institute for Plasma Research, Gandhinagar 382 428, India \\ ${ }^{b}$ Microwave Laboratory, Department of Physics, Indian Institute of Technology Delhi, New Delhi 110 016, India
}

MS received 23 September 2013; revised 18 December 2013

\begin{abstract}
Functionally graded tungsten-copper bimetallic compact with fine microstructure and good mechanical property has been synthesized by employing microwave heating method at a temperature of $800{ }^{\circ} \mathrm{C}$ and in a short processing time of $30 \mathrm{~min}$. Scanning electron microscopy and energy dispersive $\mathrm{X}$-ray analysis revealed the graded structure of synthesized sample. The fine microstructure of tungsten in each layer is caused by arrested grain growth because of the short sintering time. The overall relative density of the W/Cu functionally graded sample has reached $87 \%$ of the theoretical density. Vickers microhardness measurements, across the length of a compact, show increase in hardness value of the sample with the increase in tungsten content. The experimental hardness values match well with the theoretically calculated hardness values.
\end{abstract}

Keywords. Microwave processing; powder metallurgy; hardness measurement.

\section{Introduction}

In 1972, Bever and Duwez (1972) recognized the usefulness of functionally graded materials and later these materials have been used for thermal management in aerospace structures and fusion reactors. Functionally graded material (FGM) can be classified under a class of advanced composites that have varying microstructure across the length, area or volume and correspondingly different mechanical properties in different layers. FGM is often employed for elimination of hurdles that arise from the joining of two dissimilar materials that have different mechanical properties, such as hardness, toughness, thermal and residual stresses, etc. (Larsson and Oden 2004). Tungsten-copper FGM has unique application as part of plasma facing components (PFCs) in future fusion devices (Itoh et al 1996). PFCs in divertor regime of ITER, like tokamak, receive high heat loads $\left(>10 \mathrm{MW} / \mathrm{m}^{2}\right)$, and comprise of pure tungsten as plasma facing material and copper alloy as a heat sink material. Tungsten has the advantage of low or negligible sputtering, high strength, low tritium retention, high corrosion resistance, and the highest melting point $\left(340{ }^{\circ} \mathrm{C}\right)$, whereas copper alloy is selected because of its very good thermal conductivity, which is essential for efficient heat removal (Pintsuk and Uytdenhouwen 2010). However, joining of tungsten to copper is a challenging task because of the large mismatch of coefficients of thermal expansion $(\alpha)$ of the two materials $\left(\alpha_{\mathrm{W}}=4 \times 10^{-6} /{ }^{\circ} \mathrm{C}, \alpha_{\mathrm{Cu}}=18 \times 10^{-6} /{ }^{\circ} \mathrm{C}\right)$ (Khirwadkar et al 2011). For minimization of large thermal

\footnotetext{
*Author for correspondence (dubecharu@gmail.com)
}

stress during joining of $\mathrm{W}$ and $\mathrm{Cu}, \mathrm{W} / \mathrm{Cu}$-based FGM can be used as an intermediate layer between pure tungsten and copper alloy. With this approach cracking, delamination, etc. can be controlled, which could otherwise lead to large difference in coefficients of thermal expansion and mismatch in elastic moduli ( $\left.E_{\mathrm{Cu}} \approx 0 \cdot 2 E_{\mathrm{W}}\right)$, where $E$ stands for Young's modulus.

There are various methods for fabrication of FGMs, which include powder metallurgical processes, plasma spraying, infiltration method, centrifugal casting, spark plasma sintering, physical vapour deposition, chemical vapour deposition, electrophoretic deposition, colloidal processing, etc (Pintsuk et al 2003). In the present work, we have chosen microwave (MW) heating method for the fabrication of W/Cu FGM. During MW heating, entire mass of a material absorbs electromagnetic radiation at the same time, and can convert it into heat via dielectric, electric, magnetic, and/or eddy current loss mechanisms. MW processing is attractive because it has many unique features such as radically enhanced reaction kinetics, substantial enhancement in sinterability, rapid joining of different materials (ceramic to metal, metal to metal, etc), high reaction yields, structural uniformity of products, accelerated nucleation rates, etc. It is rapid, energy efficient and green technique for the synthesis of materials (Mukhopadhyay et al 2001; Dube et al 2009a, b). It results in energy savings as high as $90 \%$ over the conventional methods, rapid heating rates, fine microstructures, and hence, improved mechanical properties (Sutton 1992; Dube et al 2013). The rapid heating rate and short sintering time inhibit the grain growth and thereby samples retain their fine microstructure. The samples with finer microstructure are 
expected to have the higher irradiation resistance as well, which is desired for PFCs in fusion devices (Wurster and Pippan 2009). Moreover, MW heating has successfully been employed for the synthesis of bulk tungsten, copper, and $\mathrm{W} / \mathrm{Cu}$ composites. MW heating method has thus proven its capability to produce bulk metals, which may find applications in various fields. Invariably, each individual metallic powder particle in metallic compact is surrounded by a dielectric oxide layer, which prevents connectivity percolation between the particles and significantly increases absorption of microwaves (Mondal et al 2010). Nonetheless, the skin depth of MW radiation in metal plays decisive role in MW heating of metallic powders. The skin depth for tungsten and copper at $2.45 \mathrm{GHz}$ is in micrometre regime (Mondal et al 2010). Therefore, tungsten and copper particles cannot efficiently absorb microwave at room temperature. To overcome this problem in poor absorbing materials, the microwave hybrid heating method is being used. Microwave hybrid heating method combines both MW and conventional heating and invariably ceramic susceptor are used to raise the temperature of specimen up to certain level. In the present work, the hybrid MW heating method has been chosen to synthesize W/Cu FGM with fine microstructure. Instead of ceramic susceptor, plasmaassisted microwave heating has been chosen to fabricate W/Cu FGM.

\section{Experimental}

Six-layered W/Cu FGM sample was synthesized by employing multimode microwave applicator. Tungsten at each layer was $0 \%, 20 \%, 40 \%, 60 \%, 80 \%, 100 \%$ by weight. Tungsten powders with an average particle size of $6 \mu \mathrm{m}$ and copper powders with an average particle size of $20 \mu \mathrm{m}$ were used as starting materials. The powders of tungsten and copper were mixed by weight fraction for each layer separately and to ensure homogeneous mixing each mixture has been ground in mortar and pestle for $1 \mathrm{~h}$ duration. The density of each layer was calculated by using the rule of mixture for composites. The appropriate mass of pre-mixed $\mathrm{W} / \mathrm{Cu}$ powders of different compositions were stacked layer-by-layer into a die steel mould, and then the stacked powders were pressed by applying pressure of $500 \mathrm{MPa}$ into a cylindrical sample of diameter $6 \mathrm{~mm}$. The compact was processed in multimode microwave applicator (shown in figure 1), operating at $2.45 \mathrm{GHz}$ in dynamic inert (Argon) ambient. The mode stirrer, attached with the multimode applicator, ensures uniform field distribution inside the applicator.

The sample was processed at temperature of $800^{\circ} \mathrm{C}$, which is less than melting temperature of copper $\left(1083^{\circ} \mathrm{C}\right)$, to eliminate gravity-induced dimensional changes such as formation of elephant foot, etc. (Olevsky et al 2000). To avoid errors in temperature measurements, the temperature was monitored using optical pyrometer Raytek MA2SC (working temperature range $350-2000^{\circ} \mathrm{C}$ ). The synthesized FGM

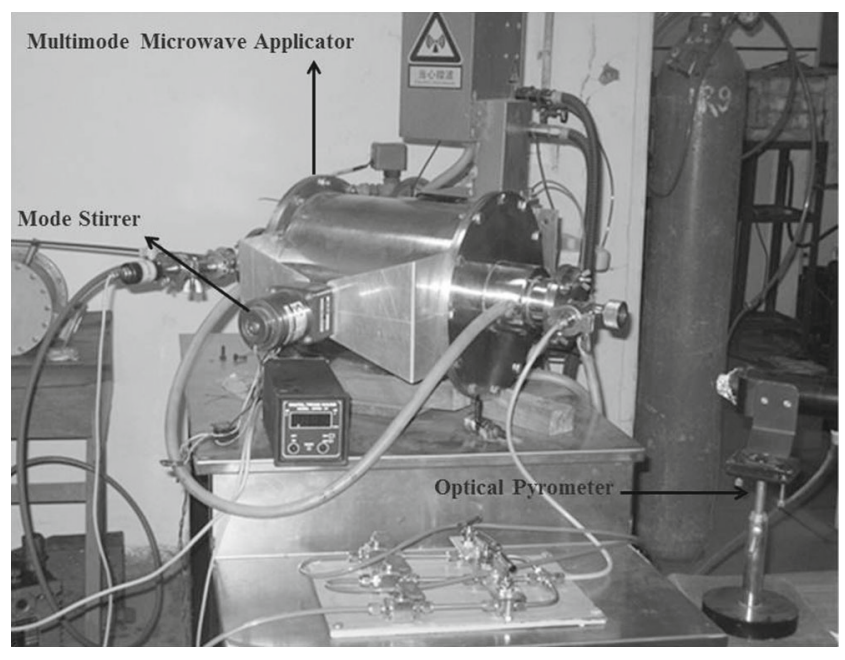

Figure 1. Multimode microwave applicator unit.

sample has been characterized for its microstructure and elemental analysis by employing scanning electron microscopy and density by Archimedes' method. The sample was gently polished starting with 600 grit $\mathrm{SiC}$ abrasive and finally with diamond paste. The variation of microhardness along the length of synthesized FGM has been measured by using a Micro-Vickers hardness tester (Mitutoyo HM211). The hardness testing machine is fitted with a diamond pyramidal indenter and attached to an optical microscope. The distance between any two indentations was kept more than two times the diagonal length of the indentation, to avoid mutual influence of the indentations. The applied load was kept $400 \mathrm{~g}$ and the time of indentation was kept constant at $10 \mathrm{~s}$ for each measurement. The diagonal of the impression was measured with a calibrated optical microscope (at $50 \times$ magnification).

\section{Results and discussion}

Initially, metallic powders will get heated mainly due to two mechanisms: (i) MW absorption by relatively smaller metallic particles, having particle size comparable with skin depth at room temperature and (ii) plasma-assisted heating. Subsequently, with the increase in temperature, metallic particles start absorbing microwaves very efficiently. Skin depth of electromagnetic radiation increases with temperature and consequently material-microwave interaction increases with temperature. At high temperatures, efficient penetration of microwaves provides sufficient volume for material-microwave interaction and hence substantial volumetric heating of metal powder takes place. In addition, particle of varying shape and size will also help in efficient material-microwave interaction, owing to generation of induced field lines (Dube et al 2013).

The synthesized FGM sample has been analysed for elemental distribution of tungsten and copper. The scanning 


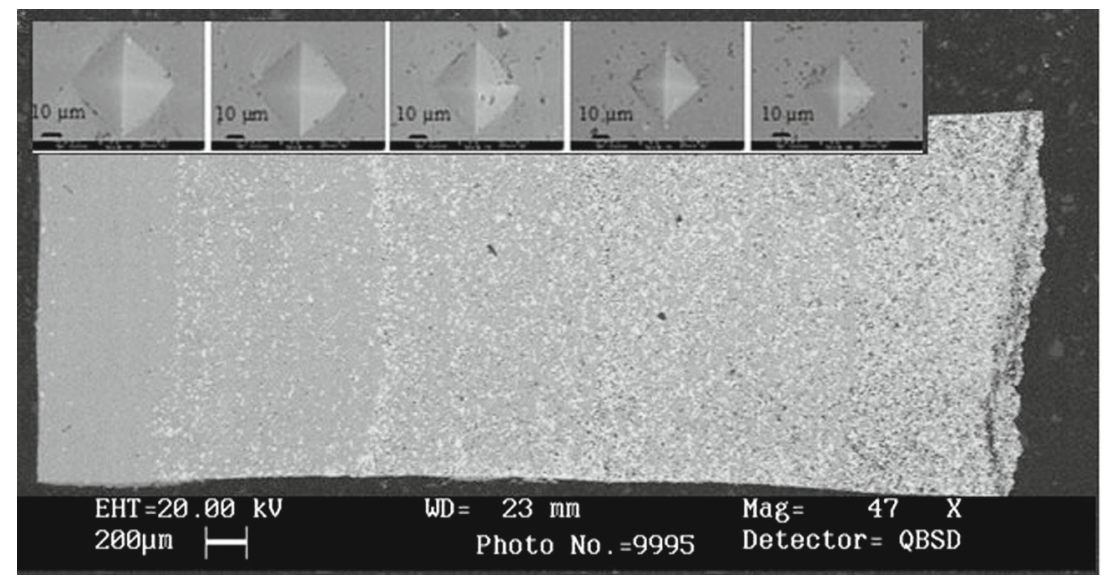

Figure 2. Scanning electron micrograph of FGM sample and indentation taken across the sample.

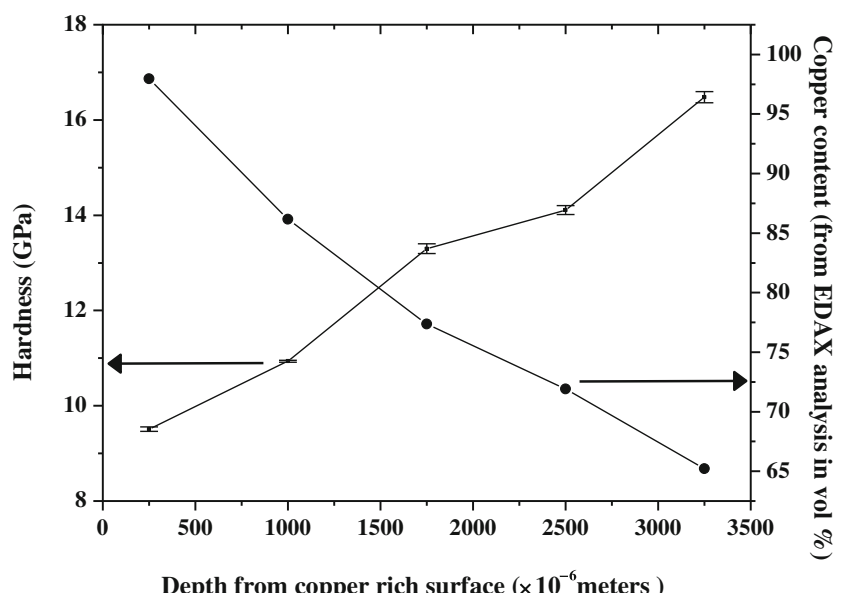

Figure 3. Hardness and elemental distribution across FGM sample.

electron micrograph (SEM) (constructed from backscattered electrons (BSEs)) of FGM sample is shown in figure 2. The tungsten has higher atomic number $(Z)$ than copper, and therefore will have greater cross-sectional area for an elastic collision with rastering electron. Consequently, the number of BSEs from tungsten will be higher, and the observed 'brighter' BSE intensity correlates with tungsten in the sample. The gradient in tungsten content across the length of compact can be seen in figure 2. Quasihomogeneous variation of tungsten content along the length of compact can be seen in the image. Moreover, the variation of tungsten along the diameter of compact is nearly uniform, which confirms one-dimensional (1D) variation of elements in the synthesized FGM sample. Therefore, the synthesized FGM sample is 1D FGM with quasihomogeneous distribution of elements, which was intended to be that way.

In order to see the gradient in mechanical properties, Vickers microhardness test has been performed across the length of FGM sample. The scanning electron images of indents in FGM sample is shown in the inset of figure 2 and it can be

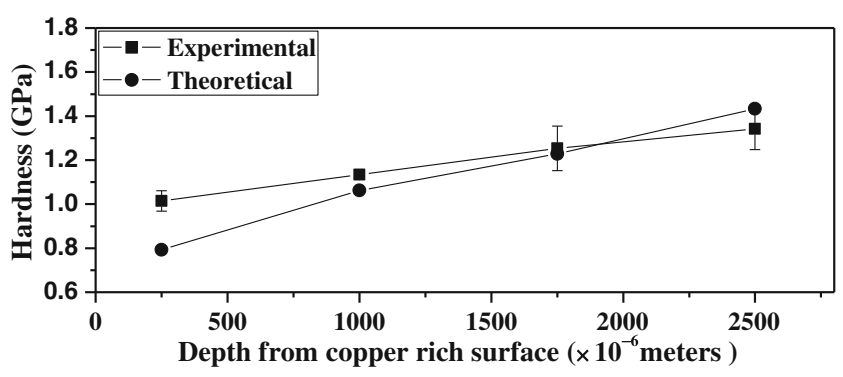

Figure 4. Experimental and theoretical hardness values of FGM sample.

seen that the indentation diameter decreases with the increase in tungsten content in the sample.

The actual elemental composition of sample near the indent position has been evaluated by EDAX analysis by selecting an area of $300 \times 300 \mu \mathrm{m}$ in between the two indents, taken along the diameter of sample. The variation of hardness and copper content along the length of FGM sample have been plotted with respect to depth from copperrich surface of FGM compact, and graphs are shown in figure 3. It may be noted from figure 3 that the hardness of FGM sample increases with the decrease in copper content/increase in tungsten content across the length of the samples. The increase in hardness with the increase in tungsten content is understandable also as the hardness value of tungsten is higher than copper. Therefore, hardness close to copper-rich surface is lower as compared with tungstenrich surface. The composite hardness was determined as a weighted average of hardness using the volume fractions of tungsten and copper, obtained from EDAX analysis. The hardness values for pure copper and pure tungsten were taken as 369 and $3430 \mathrm{MPa}$, respectively. The theoretical value of hardness, calculated from linear composite prediction rule, along with the experimental values obtained from hardness measurements is shown in figure 4 . The theoretical values of hardness and experimentally obtained hardness 


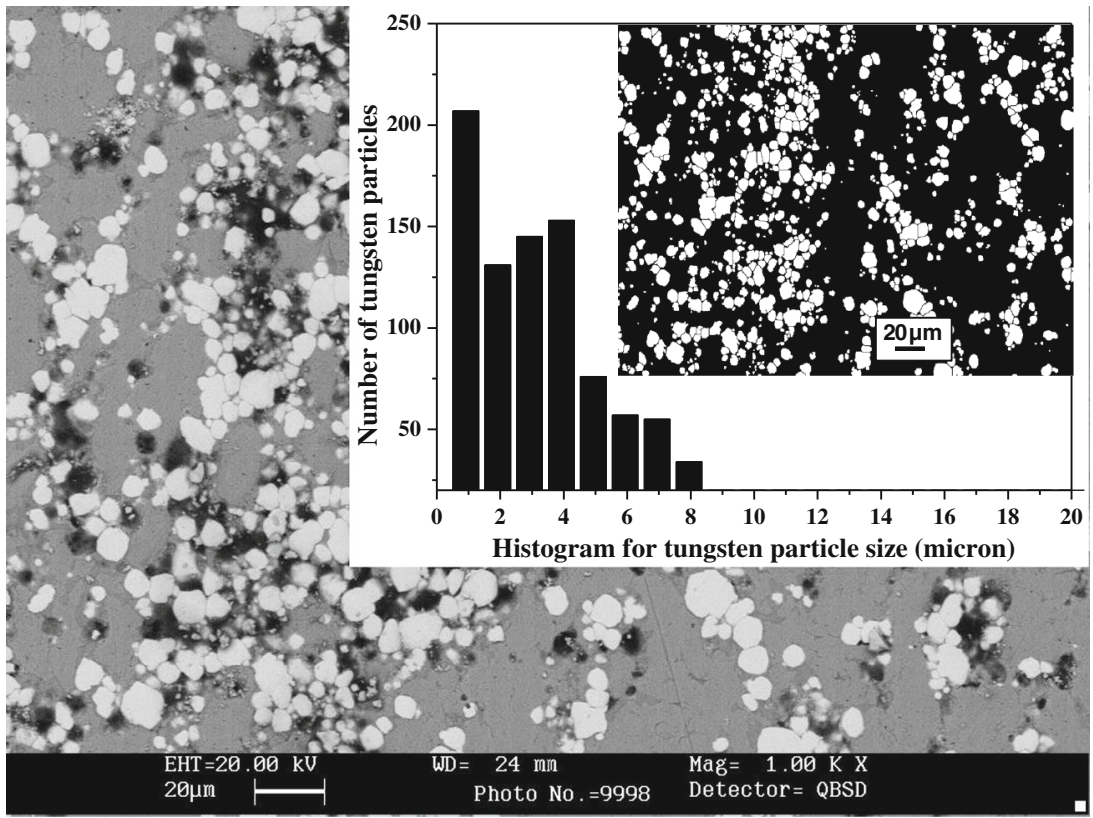

Figure 5. Scanning electron micrograph and histogram for tungsten particle size in FGM sample.

values for FGM sample are close to each other and follow a similar trend.

Subsequently, attempts have been made to correlate mechanical properties of the synthesized FGM sample with its microstructure. SEM has been taken at an arbitrary location in FGM sample and it is shown in figure 5. It can be seen in figure 5 that copper has almost reached in its molten stage at temperature of $800^{\circ} \mathrm{C}$ only, inspite of the fact that its melting temperature is nearly $1083{ }^{\circ} \mathrm{C}$. This can be attributed to the accelerated diffusion process, which occurs due to the presence of inherent non-thermal effects during microwave-material interaction (Demirskyi et al 2010; Xu et al 2012). Mass transport enchantment is well reported for microwave sintering processes (Bykov et al 2001a, b). The non-thermal interaction of electromagnetic field with material results in a higher value of diffusion coefficient during microwave heating of materials and hence an onset of melting of copper at much lower temperature is possible (Bykov et al 2001a, b). The interfacial strain may also enhance the energy of a system, which may marginally lower the melting point of copper. But in the present case lowering in melting point by $200{ }^{\circ} \mathrm{C}$, is mainly attributed to the enhanced mass transport owing to material-microwave interaction at atomic level.

The distribution of tungsten particle size in molten copper matrix has been analysed from figure 5. The SEM image has been converted to binary image, shown in the inset of figure 5, with the help of Image J program for estimation of particle size distribution of tungsten. The histogram of tungsten particle size in copper matrix has been shown in the inset of figure 5. The size of majority of tungsten particles is nearly equal to or less than $6 \mu \mathrm{m}$. Because of the short processing time and low processing temperature, the particle size of tungsten in FGM sample almost remains same as in the starting tungsten powder. Hence, microwave processing has assisted in maintaining initial fine particles of tungsten in the compact and nearly same values of theoretical and experimental hardness can be attributed to finer microstructure of FGM sample. Furthermore, the density of synthesized FGM sample has been measured by Archimedes' method and is found to be $87 \%$ of theoretical density (calculated by the rule of mixture). The inherent peculiar features of MW processing resulted in enhanced and fast densification of FGM sample at a remarkably lower processing temperature of $800{ }^{\circ} \mathrm{C}$ and in a short time span of 30 min only. Thus, it can be concluded that rapid and volumetric nature of microwave processing has resulted in good mechanical property along with the dense microstructure of W/Cu FGM sample.

\section{Conclusions}

W/Cu FGM sample has successfully been synthesized by employing hybrid microwave heating method at $800{ }^{\circ} \mathrm{C}$ and in a very short processing time of $30 \mathrm{~min}$. The SEM image and elemental distribution map of tungsten and copper across the length of compact indicate the whole graded structure of W/Cu FGM sample. Low temperature synthesis of FGM samples has become possible because of enhanced diffusion kinetics owing to non-thermal microwave effects. The short processing time has helped in maintaining the fine structure of FGM sample and thereby resulted in good mechanical property, close to theoretical value. Microwave sintering being rapid in nature rendered desired compositional gradient across the sample as well. The synthesized FGM sample 
with fine microstructure and good mechanical property can be used as an intermediate layer for fabrication PFCs in fusion devices.

\section{References}

Bever M B and Duwez P F 1972 Mater. Sci. Eng. 101

Bykov Y V, Rybakov K I and Semenov V E 2001a J. Phys. D: Appl. Phys. 34 R55

Bykov Y V, Egorov S V, Eremeev A G, Rybakov K I, Semenov V E and Sorokin A A 2001b J. Mater. Sci. 36131

Demirskyi D, Agrawal D K and Ragulya A 2010 Mater. Lett. 64 1433

Dube C L, Kashyap S C, Dube D C and Agarwal D K 2009a Appl. Phys. Lett. 94 213107-1

Dube C L, Kashyap S C, Dube D C and Agarwal D K 2009b J. Alloys Compd. $\mathbf{4 8 8} 328$

Dube C L, Kashyap S C, Dube D C and Agarwal D K 2013 J. Alloys Compd. $\mathbf{5 7 1} 75$
Itoh Y, Takahashi M and Takano H 1996 Fusion Eng. Des. 31279 Khirwadkar S S, Singh K P, Patil Y, Khan M S, Buch J J U, Patel A, Tripathi S, Jaman P M, Rangaraj L and Divakar C 2011 Fusion Eng. Des. 861736

Larsson C and Oden M 2004 Mater. Sci. Eng. A 382141

Mondal A, Agrawal D and Upadhyaya A 2010 J. Microwave Power Electromagn. Energy $\mathbf{4 4} 28$

Mukhopadhyay A K, Chaudhuri M R, Seal A, Dalui S K, Banerjee M and Phani K K 2001 Bull. Mater. Sci. 24125

Olevsky E A, German R M and Upadhyaya A 2000 Acta Mater. 48 1167

Pintsuk G and Uytdenhouwen I 2010 Int. J. Refrac. Met. Hard Mater. 28661

Pintsuk G, Brunings S E, Doring J E, Linke J, Smid I and Xue L 2003 Fusion Eng. Des. 66237

Sutton W 1992 Mater. Res. Soc. Symp. Proc. 2693

Wurster S and Pippan R 2009 Scr. Mater. 601083

Xu F, Li Y, Hu X, Niu Y, Zhao J and Zhang Z 2012 Mater. Lett. 67162 